\title{
MRNA-derived Prostate Cancer Vaccine CV9104
}

National Cancer Institute

\section{Source}

National Cancer Institute. mRNA-derived Prostate Cancer Vaccine CV9104. NCI

Thesaurus. Code C106231.

A prostate cancer vaccine containing six messenger RNAs (mRNAs) encoding for antigens upregulated in prostate cancer, including mRNAs for prostate specific antigen (PSA), prostate specific membrane antigen (PSMA), prostatic acid phosphatase (PAP), and mucin 1 (MUC1), with potential antineoplastic and immunomodulating activities. Upon intradermal administration of mRNA-derived prostate cancer vaccine CV9104, this agent enters cells, the mRNAs are translated into the respective prostate specific antigens and may cause the immune system to mount a cytotoxic T lymphocyte response $(C T L)$ against PSA-, PSMA-, PAP- and MUC1-expressing prostate tumor cells. The mRNAs used in this vaccine are modified to have enhanced translational potency and adjuvant activities. PSA, PSMA, PAP and MUC1 are frequently upregulated in prostate cancer cells; their expression in prostate cancer has been correlated with disease progression. 